\title{
Modeling of tuning of microresonator filters by perturbational evaluation of cavity mode phase shifts
}

\author{
K. R. Hiremath, Member, IEEE, and M. Hammer
}

\begin{abstract}
Microresonator filters, realized by evanescent coupling of circular cavities with two parallel bus waveguides, are promising candidates for applications in dense wavelength division multiplexing. Tunability of these filters is an essential feature for their successful deployment. In this paper we present a framework for modeling of tuning of the microresonators by changes of their cavity core refractive index. Using a reciprocity theorem, a perturbational expression for changes in the cavity propagation constants due to slight modifications of the cavity core refractive index is derived. This expression permits to analytically calculate shifts in spectral response of the 2D resonators. Comparisons of the resultant shifts and spectra with direct simulations based on coupled mode theory show satisfactory agreement.
\end{abstract}

Index Terms-Integrated Optics, numerical modeling, tuning, bent waveguides, microresonators, coupled mode theory, optical filters, whispering gallery modes

\section{INTRODUCTION}

$\mathbf{H}$ IGH Q microcavity resonators are extensively investigated for a variety of applications like lasers, sensors, the study of quantum electrodynamics, or integrated optical communication devices [1]. When such cavities in the form of circular rings or disks are coupled to single/dual bus waveguides they act as wavelength filters. Due to high Q and compactness of these filters, they are explored for dense wavelength division multiplexing in integrated optics [2], [3], [4]. The realization and actual performance of the resonators are constrained by several factors, e.g. an accurate definition of the resonance wavelengths requires a high degree of control of the geometrical parameters, temperature dependent changes in the material parameters detune the spectral response. Active (e.g. electro/thermo-optical, photobleaching) tuning of the resonators greatly relaxes these constraints [5], [6], |7]|. This controllability is also utilized in other devices like lasers, optical switches, optical modulators [8].

In essence, an active tuning is equivalent to a controllable perturbation. This perturbational viewpoint is often employed for microresonators based bio, chemo-sensors [9], [10]. Due to the sensitivity of whispering gallery modes (WGMs) of the resonators towards the environment in which they are built up, any slight change in the environment -in the exterior or the interior of the cavity- results in a shift of the resonance wavelengths, and a change of output light intensity at a fixed

K. R. Hiremath is with the Center for Optoelectronics and Optical Communications, University of North Carolina at Charlotte, Charlotte, NC 28223 USA. e-mail: khiremat@uncc.edu

M. Hammer is with the MESA ${ }^{+}$Institute for Nanotechnology, Department of Applied Mathematics, University of Twente, 7500 AE Enschede, the Netherlands. email: m.hammer@math.utwente.nl wavelength. Such wavelength shifts have been analyzed using arguments based on energy perturbations [11], [12] or rigorous finite element simulations [13]. Here we broaden these studies in the context of add/drop filters.

For the application of microresonator elements as tunable wavelength filters, suitable materials are introduced that permit a slight change of the refractive index of the cavity core by external mechanisms like electro- or thermo-optic effects. For the modeling of such tuning, in this contribution we propose and evaluate perturbational expressions for phase shifts of the modes of the bent waveguides that constitute the cavity. Similar expressions for the induced changes of propagation constants of modes supported by straight waveguides are well known [14]. We use reciprocity techniques for the derivation. When applied to given cavity modes of a resonator configuration, these phase shift expressions allow to evaluate analytically the wavelength tuning range for the respective resonances. These expressions resemble those for the frequency shifts of whispering gallery resonances of circular (uncoupled, isolated) cavities, obtained by energy perturbation arguments [15].

In principle, the proposed theoretical framework is applicable for both 3D and 2D settings. Subject to availability of the cavity (bent) modes, analytic evaluation of the shifts is also possible. Due to an easy access to 2D analytical bent modes [16], in this paper numerical results are discussed for the 2D geometry. Further we discuss use of the perturbational expressions in combination with the semi-analytical 2D model for circular microresonators, which is based on a spatial frequency-domain coupled mode theory [17]. Preliminary studies can be found in Refs. [18], [19]. Within certain limits, the phase-shift formulas permit to predict directly how the tuning affects the entire wavelength spectrum. Extension to 3D resonators is outlined in the concluding remarks.

\section{TUNING OF MICRORESONATORS}

The resonators under consideration consist of ring- or diskshaped dielectric cavities, evanescently coupled to two parallel straight bus waveguides, as illustrated in Fig. 11 The core layer of the cavities is assumed to be an active region, e.g. heaters or electrodes are placed on top of it for tuning. We consider a 2D geometry in the frequency domain setting, where a time harmonic optical signal $\exp (i \omega t)$ of given real frequency $\omega$, corresponding to vacuum wavelength $\lambda$ and wave number $k=2 \pi / \lambda$, is present everywhere. Cartesian coordinates $x$, $z$, and polar coordinates $r, \theta$ are introduced for the spatially 2D description as shown in Fig. 11 The entire structure and the 
TE- or TM-polarized optical fields are assumed to be constant in the $y$-direction.

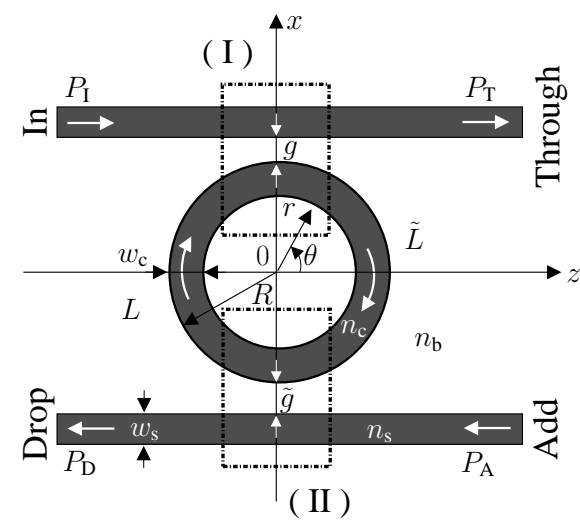

Fig. 1. Schematic microresonator representation: A cavity of radius $R$, core refractive index $n_{c}$ and width $w_{\mathcal{c}}$ is placed between two straight waveguides with core refractive index $n_{S}$ and width $w_{S}$, with gaps of widths $g$ and $\tilde{g}$ between the cavity and the bus waveguides. $n_{b}$ is the background refractive index. Tuning is applied to the core of the cavity.

To compute the spectral response, we apply a coupled mode theory (CMT) based model of the resonators [17. In this model, the resonator is represented in terms of two bentstraight waveguide couplers, I and II as in Fig. 11 which are internally connected by cavity segments of length $L$ and $\tilde{L}$ (this length is measured outside the coupler regions). The responses of the couplers I and II are characterized by their respective scattering matrices $\mathrm{S}, \tilde{\mathrm{S}}$; whereas the fields in the cavity segments are characterized by their mode propagation constants $\gamma$. Due to the leaky nature of these modes, the propagation constants are complex valued, denoted as $\gamma=\beta-\mathrm{i} \alpha$, where $\beta$ and $\alpha$ are the real valued phase propagation and attenuation constants.

Given input powers $P_{\mathrm{I}}$ and/or $P_{\mathrm{A}}$, the through power $P_{\mathrm{T}}$ and the drop power $P_{\mathrm{D}}$ can be calculated in terms of $\mathrm{S}, \tilde{\mathrm{S}}$, and $\gamma$. The computation of the spectral response can be sped up using interpolation [17], where instead of the scattering matrices $\mathrm{S}, \tilde{\mathrm{S}}$, which are associated with the couplers defined over a larger $z$ interval, one uses "reduced" scattering matrices $S^{\prime}, \tilde{S}^{\prime}$ associated with couplers of a zero length, such that the length of the cavity is $L_{\mathrm{cav}}=2 \pi R$. We use this technique for the subsequent simulations in Section IV

As explained in Refs. [18], [20], at resonance the condition $\beta=(2 m \pi+\phi) / L_{\mathrm{cav}}=\beta_{m}$ holds for the cavity mode phase propagation constant $\beta$ (real part of $\gamma$ ), where the integer $m$ gives the order of the resonance, and $\phi$ is the total phase contribution due to the coupling. Assume that the wavelength dependence of the phase constant $\beta=\beta(\lambda)$ is given. Then one can write $\beta\left(\lambda_{m}\right)=\beta_{m}$, where $\lambda_{m}$ is the resonance wavelength associated with the resonant cavity mode propagation constant $\beta_{m}$.

In principle, the tuning affects both the coupler response and the cavity mode propagation. If the coupler length is short enough, then as a first approximation one can disregard the influence of tuning on the couplers, and assume that a tuning mechanism, modeled by a parameter $p$, affects mainly the wave propagation along the cavity. We verify the validity of this approximation for the subsequent simulations in Section IV where it is used to simplify the computations. Now besides the wavelength, the cavity mode propagation constant also depends on the tuning parameter, i.e. $\beta=\beta(p, \lambda)$, with $p=0$ representing the original state: $\beta\left(0, \lambda_{m}\right)=\beta_{m}$.

As a result of tuning, the resonance of order $m$ is shifted towards a new wavelength $\tilde{\lambda}_{m}$, such that $\beta\left(p, \tilde{\lambda}_{m}\right)=(2 m \pi+$ $\phi) / L_{\text {cav }}=\beta_{m}$ is satisfied again, i.e. the wavelength shift compensates the change in the cavity mode propagation constant due to a nonzero perturbation strength $p$. A linear approximation in the tuning parameter and in the wavelength differences leads to

$$
\beta\left(p, \tilde{\lambda}_{m}\right) \approx \beta\left(0, \lambda_{m}\right)+\left.p \frac{\partial \beta}{\partial p}\right|_{0, \lambda_{m}}+\left.\left(\tilde{\lambda}_{m}-\lambda_{m}\right) \frac{\partial \beta}{\partial \lambda}\right|_{0, \lambda_{m}} ^{\stackrel{!}{=}} \beta_{m} .
$$

Hence the shift in the resonant wavelength $\Delta_{p} \lambda_{m}=\tilde{\lambda}_{m}-\lambda_{m}$ that is effected by the tuning can be written as

$$
\Delta_{p} \lambda_{m}=-\left.p\left(\frac{\partial \beta}{\partial p}\right)\left(\frac{\partial \beta}{\partial \lambda}\right)^{-1}\right|_{0, \lambda_{m}} \text {. }
$$

Express the propagation constant in terms of vacuum wavenumber and effective mode index as $\beta=2 \pi n_{\text {eff }} / \lambda$; if the wavelength dependence of the effective index is negligible, then

$$
\frac{\partial \beta}{\partial \lambda}=-\frac{\beta}{\lambda}+k \frac{\partial n_{\mathrm{eff}}}{\partial \lambda} \approx-\frac{\beta}{\lambda} .
$$

The same approximation can also be obtained by homogeneity arguments [21] for the propagation constants; alternatively a more accurate representation of the wavelength dependence of the propagation constant in terms of the group effective index of the cavity mode can also be derived [18]. With the above approximation, the wavelength shift reads

$$
\Delta_{p} \lambda_{m}=p \frac{\partial \beta}{\partial p} \frac{\lambda_{m}}{\beta_{m}} .
$$

Note that the wavelength shift does not explicitly depend on the length of the cavity.

We are interested in tuning by a slight change $\Delta n_{\mathrm{c}}$ of the cavity core refractive index. The resultant shifts in resonance wavelengths are given by

$$
\Delta \lambda_{m}=\Delta n_{\mathrm{c}} \frac{\partial \beta}{\partial n_{\mathrm{c}}} \frac{\lambda_{m}}{\beta_{m}} .
$$

In order to estimate this effect, one must know the dependence of the propagation constants on the core refractive index. In the next section we derive a perturbational expression for the change in the cavity propagation constants due to a perturbation of the core refractive index.

\section{A PERTURbATIONAL EXPRESSION FOR BEND MODE PHASE SHIFTS}

As outlined in Section II in the CMT model of the microresonators the cavity is segmented into pieces of bent waveguides. 
Eq. (5) requires an expression for the derivatives, i.e. the first order changes, of the bend mode propagation constants with respect to the cavity core refractive index. Here we adhere to the 2D setting as introduced in Fig. 1 with polar coordinates $(r, y, \theta)$ (invariance in the $y$-direction). For a bent waveguide with radial refractive index distribution $n(r)=\sqrt{\epsilon(r)}$, let the full electric $(\boldsymbol{E})$ and magnetic $(\boldsymbol{H})$ field for a given mode be

$$
\left(\begin{array}{c}
\boldsymbol{E} \\
\boldsymbol{H}
\end{array}\right)(r, \theta, t)=\left(\begin{array}{c}
\left(\tilde{E}_{r}, \tilde{E}_{y}, \tilde{E}_{\theta}\right) \\
\left(\tilde{H}_{r}, \tilde{H}_{y}, \tilde{H}_{\theta}\right)
\end{array}\right)(r) \mathrm{e}^{\mathrm{i}(\omega t-\gamma R \theta),}
$$

where the $\sim$ symbol indicates the mode profile. These mode profiles and the corresponding propagation constants $\gamma$ of the bent waveguides are computed analytically according to the expressions given in Ref. [16].

Suppose that the core refractive index is slightly perturbed, and the perturbed refractive index distribution is given by $n_{p}(r)=$ $\sqrt{\epsilon_{p}(r)}$. Assuming that the mode profile remains unchanged for this perturbation, the corresponding perturbed modal field $\left(\boldsymbol{E}_{p}, \boldsymbol{H}_{p}\right)$ is approximated as

$$
\left(\begin{array}{c}
\boldsymbol{E}_{p} \\
\boldsymbol{H}_{p}
\end{array}\right)=P(\theta)\left(\begin{array}{c}
\boldsymbol{E} \\
\boldsymbol{H}
\end{array}\right)
$$

where $P(\theta)$ is an unknown function of the angular coordinate $\theta$.

By applying Lorentz's reciprocity theorem [14] in polar coordinates to $\left(\boldsymbol{E}_{p}, \boldsymbol{H}_{p}, \epsilon_{p}\right)$ and $(\boldsymbol{E}, \boldsymbol{H}, \epsilon)$, one obtains

$$
\begin{aligned}
\int_{0}^{\infty} \nabla \cdot\left(\boldsymbol{E}_{p} \times \boldsymbol{H}^{*}\right. & \left.+\boldsymbol{E}^{*} \times \boldsymbol{H}_{p}\right) r \mathrm{~d} r \\
& =-\mathrm{i} \omega \epsilon_{0} \int_{0}^{\infty}\left(\epsilon_{p}-\epsilon\right) \boldsymbol{E}_{p} \cdot \boldsymbol{E}^{*} r \mathrm{~d} r
\end{aligned}
$$

which upon inserting the ansatz (7) and after simplifying reduces to

$$
\begin{aligned}
\frac{d P}{d \theta} \int_{0}^{\infty} \boldsymbol{a}_{\theta} \cdot\left(\boldsymbol{E} \times \boldsymbol{H}^{*}+\boldsymbol{E}^{*} \times \boldsymbol{H}\right) \mathrm{d} r & \\
& =-\mathrm{i} \omega \epsilon_{0} P \int_{0}^{\infty}\left(\epsilon_{p}-\epsilon\right) \boldsymbol{E} \cdot \boldsymbol{E}^{*} r \mathrm{~d} r
\end{aligned}
$$

where $\boldsymbol{a}_{\theta}$ is the unit vector in the angular direction. Inserting the bent waveguide field ansatz (6) and solving for $P(\theta)$ leads to

$P(\theta)=P_{0} \exp \left(-\mathrm{i} \omega \epsilon_{0} \frac{\int_{0}^{\infty}\left(\epsilon_{p}-\epsilon\right) \tilde{\boldsymbol{E}} \cdot \tilde{\boldsymbol{E}}^{*} r \mathrm{~d} r}{\int_{0}^{\infty} \boldsymbol{a}_{\theta} \cdot\left(\tilde{\boldsymbol{E}} \times \tilde{\boldsymbol{H}}^{*}+\tilde{\boldsymbol{E}}^{*} \times \tilde{\boldsymbol{H}}\right) \mathrm{d} r} \theta\right)$,

where $P_{0}$ is a constant, the superscript $\sim$ represents the mode profile. Thus the perturbed modal field is

$$
\left(\begin{array}{c}
\boldsymbol{E}_{p} \\
\boldsymbol{H}_{p}
\end{array}\right)=P_{0}\left(\begin{array}{c}
\tilde{\boldsymbol{E}} \\
\tilde{\boldsymbol{H}}
\end{array}\right) \exp (-\mathrm{i}(\gamma+\delta \gamma) R \theta),
$$

and the change in propagation constant $\delta \gamma$ due to the perturbation is given by

$$
\delta \gamma=\frac{\omega \epsilon_{0}}{R} \frac{\int_{0}^{\infty}\left(\epsilon_{p}-\epsilon\right) \tilde{\boldsymbol{E}} \cdot \tilde{\boldsymbol{E}}^{*} r \mathrm{~d} r}{\int_{0}^{\infty} \boldsymbol{a}_{\theta} \cdot\left(\tilde{\boldsymbol{E}} \times \tilde{\boldsymbol{H}}^{*}+\tilde{\boldsymbol{E}}^{*} \times \tilde{\boldsymbol{H}}\right) \mathrm{d} r} .
$$

Note that the above expression can also be written in terms of modal fields $(\boldsymbol{E}, \boldsymbol{H})$ instead of mode profiles $(\tilde{\boldsymbol{E}}, \tilde{\boldsymbol{H}})$. Then it is evident that the denominator of the fraction on the right hand side of Eq. 10 is equal to $4 P_{\theta}(\theta)$, where $P_{\theta}(\theta)$ is the power transported by the (unperturbed) bent mode in the angular direction [16]. Thus for a mode normalized to unit modal power, the change in propagation constant is directly proportional to the strength of $\int_{0}^{\infty}\left(\epsilon_{p}-\epsilon\right) \tilde{\boldsymbol{E}} \cdot \tilde{\boldsymbol{E}}^{*} r \mathrm{~d} r$, i.e. the shift is the largest if the permittivity perturbation is present at radial positions, where the squared bent mode profile $\left(|\boldsymbol{E}|^{2}=\boldsymbol{E} \cdot \boldsymbol{E}^{*}\right)$ is strong. For typical well guided modes supported by a cavity ring, a permittivity perturbation of the core layer automatically fulfills this requirement. In case of whispering gallery modes (WGMs) of disk-shaped resonators, the field maximum is in the vicinity of the outer dielectric layer interface. A slight perturbation in that region is immediately picked up by the WGMs. Precisely this sensitivity of the modes is utilized in microcavity based sensors. One can see a close formal resemblance of Eq. 10 for the change in the cavity propagation constant to the expression for the shift of WGMs in microspheres by protein adsorption given in Ref. [15].

The right hand side of Eq. 10 is a pure real number. Therefore this expression, in fact, gives the change in the real part of the propagation constant only, denoted by $\delta \beta$. In Ref. [14] a similar expression for the change in the propagation constant for bulk uniform permittivity perturbations of straight waveguides is derived by means of a variational principle.

In the present case of bent waveguides, the use of an asymptotic expansion of Hankel functions of second kind $\mathrm{H}_{\nu}^{(2)}(n k r)$ (see Ref. [16]) reveals that, if $\epsilon_{p}-\epsilon$ does not vanish for large radial coordinates, the integral $\int_{0}^{\infty}\left(\epsilon_{p}-\epsilon\right) \tilde{\boldsymbol{E}} \cdot \tilde{\boldsymbol{E}}^{*} r \mathrm{~d} r$ is undefined for the upper limit $r=\infty$ (obviously the template 6 for the perturbed field does not constitute an acceptable approximation in that case of a uniform alteration of the properties of the exterior cladding). Still, for a radially bounded perturbation $\delta \epsilon_{\mathrm{c}}=\delta n_{\mathrm{c}}^{2}=n_{\mathrm{c} p}^{2}-n_{\mathrm{c}}^{2}$ of the core refractive index, Eq. 10 is well defined; in that case it simplifies to

$$
\delta \beta=\frac{\omega \epsilon_{0}}{R} \frac{\delta n_{\mathrm{c}}^{2} \int_{R-w_{c}}^{R} \boldsymbol{E} \cdot \boldsymbol{E}^{*} r \mathrm{~d} r}{\int_{0}^{\infty} \boldsymbol{a}_{\theta} \cdot\left(\boldsymbol{E} \times \boldsymbol{H}^{*}+\boldsymbol{E}^{*} \times \boldsymbol{H}\right) \mathrm{d} r}
$$

where $R-w_{c}$ and $R$ define the core interfaces as shown in Fig. 11 and $n_{\mathrm{c} p}$ and $n_{\mathrm{c}}$ are the perturbed and unperturbed core refractive index respectively. For a small perturbation one can approximately write

$$
\begin{aligned}
\frac{\partial \beta}{\partial n_{\mathrm{c}}} & =2 n_{\mathrm{c}} \frac{\partial \beta}{\partial \epsilon_{\mathrm{c}}} \approx 2 n_{\mathrm{c}} \frac{\delta \beta}{\delta \epsilon_{c}} \\
& =2 n_{\mathrm{c}} \frac{\omega \epsilon_{0}}{R} \frac{\int_{R-w_{c}}^{R} \boldsymbol{E} \cdot \boldsymbol{E}^{*} r \mathrm{~d} r}{\int_{0}^{\infty} \boldsymbol{a}_{\theta} \cdot\left(\boldsymbol{E} \times \boldsymbol{H}^{*}+\boldsymbol{E}^{*} \times \boldsymbol{H}\right) \mathrm{d} r}
\end{aligned}
$$

Note that the integrals that occur in the above expression are well behaved. Here $\boldsymbol{E}, \boldsymbol{H}$ are the electric field and magnetic field of the cavity mode associated with the $m$ 'th order unperturbed (untuned) resonance. Using this expression with Eq. [5), gives desired wavelength shift due to tuning. 


\section{Simulation RESUlts}

First we assess the validity of the perturbation expression (12). For the moderately lossy bent waveguide configuration considered in Fig. 2 the estimation of the change in the phase propagation constants by the perturbation expression agrees very well with the values computed directly by the analytical bent waveguide model.

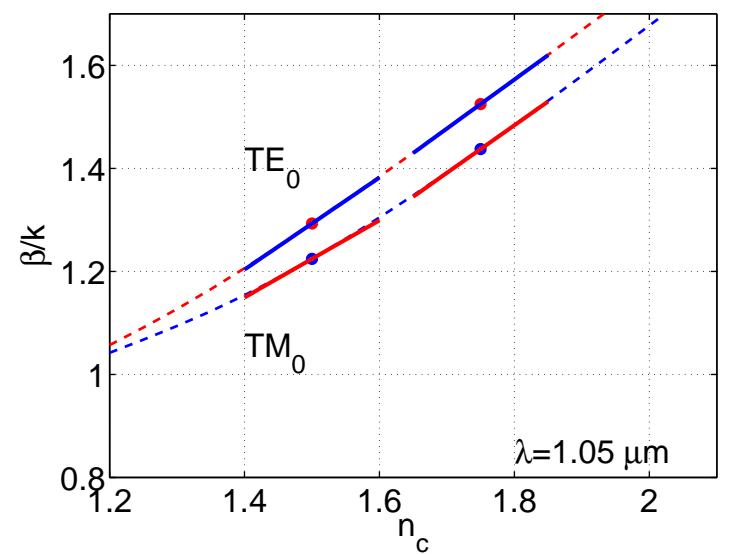

Fig. 2. Phase propagation constants estimated by the perturbational expression, for a bent waveguide configuration with width $w_{c}=0.5 \mu \mathrm{m}, R=5 \mu \mathrm{m}$ and uniform background $n_{b}=1$. Dashed lines denote $\beta / k$ obtained by direct calculations [16], dots are reference points $n_{c}=1.5$ and $n_{c}=1.75$, and the slope of the solid line segments is given by expression 12 .

As an another example, for Fig. 3 the perturbational expression 12 is evaluated for WGMs supported by a single curved interface (meant as piece of a resonator disk). For the moderately lossy fundamental and first order WGMs, the agreement is excellent, but for the second order WGMs which are considerably lossy (e.g. $n_{\mathrm{c}}=1.5, \gamma / k=1.0422-\mathrm{i} 5.7410 \cdot 10^{-3}$ $\left.\left(\mathrm{TE}_{2}\right), 1.0339-\mathrm{i} 1.21610 \cdot 10^{-2}\left(\mathrm{TM}_{2}\right)\right)$ there are major deviations. Apparently, here the changes in the mode profiles and the attenuation constants due to the core refractive index perturbation are not negligible, such that the ansatz (7) is not appropriate for these fields.

Having assessed the expression (12, now we validate the resonance shifts predicted by Eq. (5) using Eq. (12). Table I gives comparison for the shifts for the test case of the ring resonator. For the present perturbation, it is evident that the shifts predicted by using Eq. (5) using the perturbational expression (12) agree satisfactorily with direct CMT simulations.

\begin{tabular}{|c|c|c|}
\hline$\lambda_{m} \mu \mathrm{m}(\mathrm{CMT})$ & $\Delta \lambda_{m} \mu \mathrm{m}$ (Eq. [5, 12) $)$ & $\Delta \lambda_{m} \mu \mathrm{m}$ (CMT) \\
\hline 1.0184 & 0.0025 & 0.0025 \\
\hline 1.0413 & 0.0026 & 0.0027 \\
\hline 1.0654 & 0.0026 & 0.0027 \\
\hline
\end{tabular}

TABLE I

Comparison of ring resonator TE mode spectral shifts $\Delta \lambda_{m}$ predicted by

Eq. 5] (second column) with direct calculations (third column) for a structure according to Fig. 1] with $R=5 \mu \mathrm{m}, w_{\mathrm{c}}=w_{\mathrm{s}}=0.3 \mu \mathrm{m}, n_{\mathrm{s}}=1.5$,

$n_{b}=1.0, g=\tilde{g}=0.2 \mu \mathrm{m}$. The cavity core refractive index is $n_{c}=1.5$ for the unperturbed setting, and $n_{c p}=1.504$ for the perturbed structure.

For the evaluation of the effect of tuning, in principle one can compute the resonator spectra for the unperturbed and
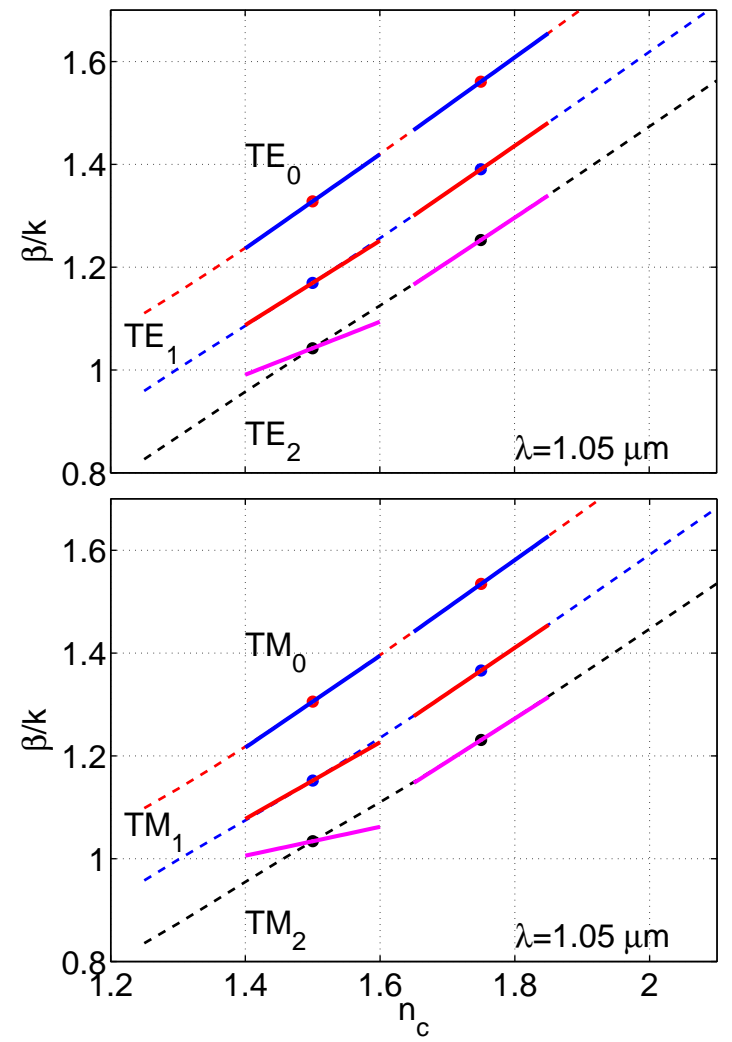

Fig. 3. Phase propagation constants of WGMs evaluated by the perturbational expression [12, for a bent waveguide configuration with $n_{b}=1.0, w_{c}=$ $R=5 \mu \mathrm{m}$. Interpretation of the curves is as for Fig. 2 The subscripts $0,1,2$ denote the order of the modes.

the perturbed configurations separately using the CMT based interpolation method described in Section 1 Let's assume that for a slight change of the cavity core refractive index the coupler scattering matrices $S^{\prime}, \tilde{S}^{\prime}$ do not change much, and the shifts of the resonances are entirely due to the changes in the cavity mode propagation constants. Then using $\mathrm{S}^{\prime}, \tilde{\mathrm{S}}^{\prime}$ of the unperturbed resonator, and adding the phase propagation constant shifts $\delta \beta$ to the propagation constants $\gamma$ of the unperturbed cavity segments, one can again follow the previously described interpolation method, without recalculating the scattering matrices for the perturbed resonator. In this way, a significant amount of computational work can be avoided. We will verify this approach.

Fig. 4 depicts the spectral responses for the perturbed and the unperturbed resonators. As seen in the top plot, for the ring resonator the spectral response computed with the above approximation method (solid line) agrees quite well with the direct CMT calculation (circles). As far as the resonance positions are concerned, similar agreement is found also in case of the present multimodal disk resonator, as shown in the bottom plots. This agreement confirms the previous claim that the influence of moderate tuning can be reliably captured by a mere effect on the cavity mode propagation, without significant changes in the strength of the interaction with the external waveguides. For the disk resonator, minor deviations are observed in the depths of the resonance dips, in particular for the $\mathrm{TE}_{1}$ resonances, where apparently the change in modal 

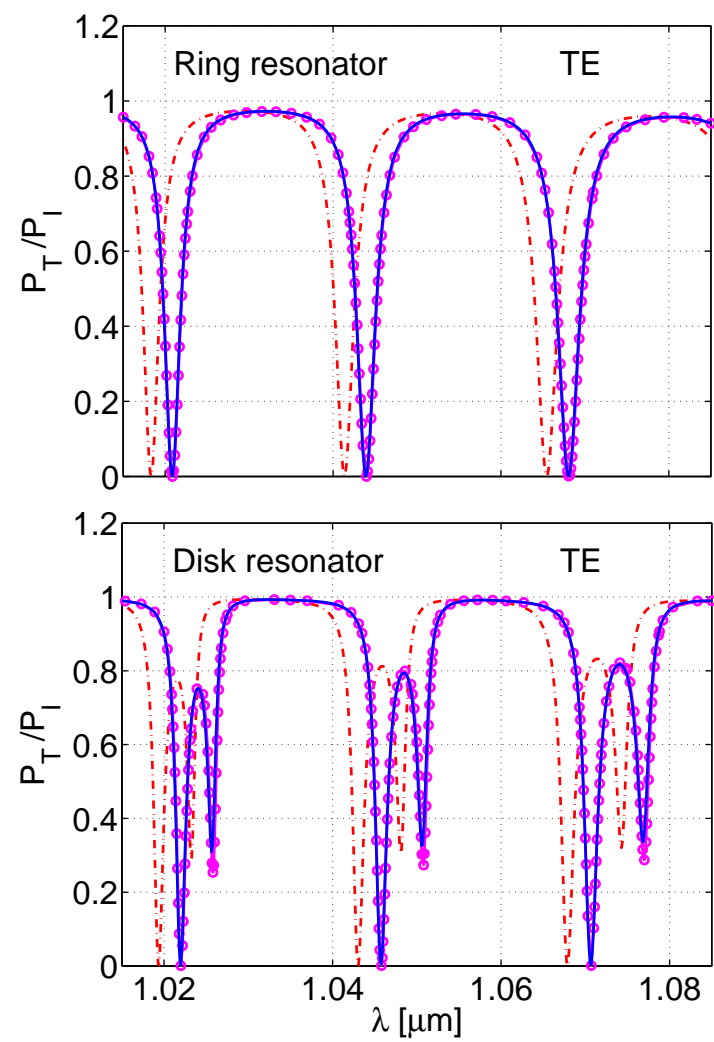

Fig. 4. Spectral shift due to tuning of the cavity core refractive index, for the ring- (top plot, $w_{c}=0.3 \mu \mathrm{m}$ ) and disk-resonator configurations (bottom plot, $\left.w_{\mathrm{c}}=R=5 \mu \mathrm{m}\right)$ of Table $\mathbb{I}$ The curves of the normalized transmitted power are calculated by the interpolation method of Ref. 17] (nodal wavelengths: $1.015 \mu \mathrm{m}, 1.05 \mu \mathrm{m}, 1.085 \mu \mathrm{m})$ for the unperturbed resonator with $n_{c}=1.5$ (dash-dotted line) and for the perturbed resonator with $n_{c p}=1.504$ (circles). Solid lines represent the results of the approximation based on the perturbation expressions as outlined in the text.

attenuation due to the core refractive index change is slightly larger than for the $\mathrm{TE}_{0}$ mode. The reason for these deviations is that the present perturbation approach does not take into account alterations in the cavity mode attenuation constants. Otherwise, the agreement is quite good.

\section{CONCLUSIONS}

In this paper we modeled the tuning of microresonator based filters by changes of their cavity core refractive index. Slight changes in the refractive index affect mainly the propagation constants of the cavity modes; the respective phase shifts can be calculated using a reciprocity technique. The formulas are applicable to uniform localized perturbations of the radial permittivity profile; complications due to nonconvergent integrands do not arise. This approach accounts only for the change in the real part of the propagation constants. Nevertheless, the spectral responses for 2D microresonators obtained with this perturbational evaluation agree quite well with direct simulations based on 2D coupled mode theory. Especially for resonances involving less lossy (fundamental) modes, the agreement for the shifts of resonance positions is very satisfactory. If more lossy (higher order) modes are involved, slight deviations in the resonant power drop are observed. Using the scattering matrices and the cavity propagation constants of the unperturbed structure in combination with the shifts in the cavity mode propagation constants given by the perturbational expression, one can reliably and efficiently predict the spectral response for moderately perturbed resonators.

Even though here the simulation results are discussed for 2D microresonators, the tuning model presented is equally applicable to $3 \mathrm{D}$ resonators. In the latter case the present integrals over the $1 \mathrm{D}$ radial cross section in Eqs. 10, 12 will become integrals over the radial/axial cross section plane of the cavity core. Bend mode phase shifts can then be evaluated on the basis of (necessarily approximate) 2D mode profiles as provided e.g. by the quasi-analytical bend mode solver of Ref. [22]. Further, using a 3D CMT model of resonators [23] one can follow similar steps as outlined here to predict the tuned spectral responses. Thus extension of the present framework to a 3D setting should be straightforward.

\section{ACKNOWLEDGMENT}

This work was carried out as a part of the project 'NAIS' (IST-2000-28018), funded by the European Commission. K. R. Hiremath also acknowledges funding by DARPA Grant No. W911NF-05-2-0053, and thanks Dr. V. N. Astratov and Prof. Dr. M. A. Fiddy for support.

\section{REFERENCES}

[1] K. Vahala, editor. Optical microcavities. World Scientific, 2004.

[2] E. A. J. Marcatili. Bends in optical dielectric guides. The Bell System Technical Journal, 2103-2132, September 1969.

[3] B. E. Little, S. T. Chu, H. A. Haus, J. Foresi, and J. P. Laine. Microring resonator channel dropping filters. IEEE Journal of Lightwave Technology, 15(6):998-1005, June 1997.

[4] M. Bertolotti, A. Driessen, and F. Michelotti, editors. Microresonators as building blocks for VLSI photonics, volume 709 of AIP conference proceedings. American Institute of Physics, Melville, New York, 2004.

[5] P. Rabiei, W. H. Steier, C. Zhang, and L. R. Dalton. Polymer microring filters and modulators. IEEE Journal of Lightwave Technology, 20(11):1968-1975, November 2002.

[6] D. J. W. Klunder, F. S. Tan, T. van der Veen, H. F. Bulthuis, G. Sengo, B. Docter, H. J. W. M. Hoekstra, and A. Driessen. Experimental and numerical study of SiON microresonators with air and polymer cladding. IEEE Journal of Lightwave Technology, 21(4):1099-1110, April 2003.

[7] J. K. S. Poon, Y. Huang, G. T. Paloczi, A. Yariv, C. Zhang, and L. R. Dalton. Wide-range tuning of polymer microring resonators by the photobleaching of CLD-1 chromophores. Optics Letters, 29(22):25842586, November 2004.

[8] R. G. Hunsperger. Integrated optics theory and technology. Springer, 4 edition, 1995.

[9] R. W. Boyd and J. E. Heebner. Sensitive disk resonator photonic biosensor. Applied Optics, 40(31):5742-5747, November 2001.

[10] S. Blair and Y. Chen. Resonant-enhanced evanescent-wave fluorescence biosensing with cylindrical optical cavities. Applied Optics, 40(4):570 582, February 2001.

[11] M. M. Mazumder, D. Q. Chowdhury, S. C. Hill, and R. K. Chang. Optical resonances of a spherical dielectric microcavity: Effect of perturbations. In R. K. Chang and A. J. Campillo, editors, Optical processes in microcavities, pages 209-256. World Scientific, 1996.

[12] I. Teraoka and S. Arnold. Theory of resonance shifts in TE and TM whispering gallery modes by nonradial perturbations for sensing applications. Journal of the Optical Society of America B, 23:1381-1389, July 2006.

[13] H. Quan and Z. Guo. Simulation of whispering-gallery-mode resonance shifts for optical miniature biosensors. Journal of Quantitative Spectroscopy and Radiative Transfer, 93:231-243, 2005.

[14] C. Vassallo. Optical Waveguide Concepts. Elsevier, Amsterdam, 1991. 
[15] S. Arnold, M. Khoshsima, I. Teraoka, S. Holler, and F. Vollmer. Shift of whispering-gallery modes in microspheres by protein adsorption. Optics Letters, 28(4):272-274, February 2003.

[16] K. R. Hiremath, M. Hammer, S. Stoffer, L. Prkna, and J. Čtyroký. Analytic approach to dielectric optical bent slab waveguides. Optical and Quantum Electronics, 37(1-3):37-61, January 2005.

[17] K. R. Hiremath, R. Stoffer, and M. Hammer. Modeling of circular integrated optical microresonators by 2-D frequency domain coupled mode theory. Optics Communications, 257(2):277-297, January 2006.

[18] M. Hammer, K. R. Hiremath, and R. Stoffer. Analytical approaches to the description of optical microresonator devices. In M. Bertolotti, A. Driessen, and F. Michelotti, editors, Microresonators as building blocks for VLSI photonics, volume 709 of AIP conference proceedings, pages 48-71. American Institute of Physics, Melville, New York, 2004.

[19] K. R. Hiremath. Coupled mode theory based modeling and analysis of circular optical microresonators. PhD thesis, University of Twente, The Netherlands, 2005.

[20] K. Okamoto. Fundamentals of Optical Waveguides. Academic Press, U.S.A, 2000.

[21] M. Lohmeyer, N. Bahlmann, O. Zhuromskyy, and P. Hertel. Perturbational estimation of geometry tolerances for rectangular integrated optics devices. In Proceedings of SPIE, Integrated Optics Devices III, volume 3620, pages 311-319, 1999.

[22] L. Prkna, M. Hubálek, and J. Čtyroký. Vectorial eigenmode solver for bent waveguides based on mode matching. IEEE Photonics Technology Letters, 16(9):2057-2059, September 2004.

[23] R. Stoffer, K. R. Hiremath, M. Hammer, L. Prkna, and J. Čtyroký. Cylindrical integrated optical microresonators: Modeling by 3-D vectorial coupled mode theory. Optics Communications, 256(1-3):46-67, December 2005 .

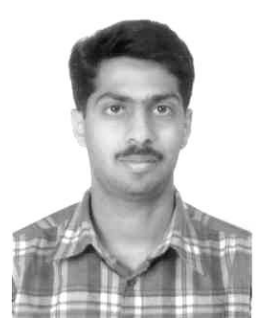

Dr. Kirankumar Rajshekhar Hiremath is a postdoctoral researcher at University of North Carolina at Charlotte in Center for Optoelectronics and Optical Communications. He has a $\mathrm{PhD}$ in Applied Mathematics from University of Twente, the Netherlands (2005), MSc in Mathematics from Indian Institute of Technology Bombay, India (1999), and BSc (Honors) in Physics from Shivaji University, Kolhapure, India (1997). His PhD dissertation was on "Coupled Mode Theory Based Modeling and Analysis of Circular Optical Microresonators". His research interests are mathematical and computational aspects of optics, modeling and simulation of optical devices, and applied mathematical analysis.

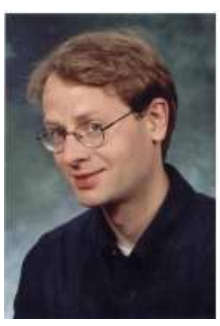

Dr. Manfred Hammer (1969, - 05.2001:

M. Lohmeyer) received the Diploma (1995) and the PhD degrees (1999) in Theoretical Physics from the University of Osnabrueck, Germany. In 2000 he joined the University of Twente, Enschede, The Netherlands, where at present he is Associate Professor in the chair Applied Analysis \& Mathematical Physics in the Department of Applied Mathematics, and in the MESA ${ }^{+}$Institute for

Nanotechnology, respectively. His research interests
are in the theoretical, numerical and semi-analytical modeling of photonic structures. 